\title{
LRRK2 Ablation Attenuates Alpha-Synuclein-Induced Neuroinflammation Without Affecting Neurodegeneration or Neuropathology In Vivo
}

\author{
Anke Van der Perren ${ }^{1}$ - Diego Cabezudo ${ }^{1} \cdot$ Géraldine Gelders $^{1} \cdot$ Javier M. Peralta Ramos ${ }^{2} \cdot$ Chris Van den Haute $^{1,3}$. \\ Veerle Baekelandt ${ }^{1} \cdot$ Evy Lobbestael $^{1}$ (D)
}

Accepted: 9 January 2021 / Published online: 16 February 2021

(C) The American Society for Experimental NeuroTherapeutics, Inc. 2021

\begin{abstract}
The development of disease-modifying therapies for Parkinson's disease is a major challenge which would be facilitated by a better understanding of the pathogenesis. Leucine-rich repeat kinase 2 (LRRK2) and $\alpha$-synuclein are key players in Parkinson's disease, but their relationship remains incompletely resolved. Previous studies investigating the effect of LRRK2 on $\alpha$-synuclein-induced neurotoxicity and neuroinflammation in preclinical Parkinson's disease models have reported conflicting results. Here, we aimed to further explore the functional interaction between $\alpha$-synuclein and LRRK2 and to evaluate the therapeutic potential of targeting physiological LRRK2 levels. We studied the effects of total LRRK2 protein loss as well as pharmacological LRRK2 kinase inhibition in viral vector-mediated $\alpha$-synucleinbased Parkinson's disease models developing early- and late-stage neurodegeneration. Surprisingly, total LRRK2 ablation or in-diet treatment with the LRRK2 kinase inhibitor MLi-2 did not significantly modify $\alpha$-synuclein-induced motor deficits, dopaminergic cell loss, or $\alpha$-synuclein pathology. Interestingly, we found a significant effect on $\alpha$ synuclein-induced neuroinflammatory changes in the absence of LRRK2, with a reduced microglial activation and $\mathrm{CD}^{+}$and $\mathrm{CD}^{+} \mathrm{T}$ cell infiltration. This observed lack of protection against $\alpha$-synuclein-induced toxicity should be well considered in light of the ongoing therapeutic development of LRRK2 kinase inhibitors for idiopathic Parkinson's disease. Future studies will be crucial to understand the link between these neuroinflammatory processes and disease progression as well as the role of $\alpha$-synuclein and LRRK2 in these pathological events.
\end{abstract}

Key Words Leucine-rich repeat kinase $2 \cdot$ alpha-synuclein $\cdot$ Parkinson's disease $\cdot$ neuroinflammation $\cdot$ kinase inhibition

Anke Van der Perren et al., Diego Cabezudo, Veerle Baekelandt and EvyLobbestael contributed equally to this work.

Veerle Baekelandt

veerle.baekelandt@kuleuven.be

Evy Lobbestael

evy.lobbestael@kuleuven.be

1 Laboratory for Neurobiology and Gene Therapy, Department of Neurosciences, Leuven Brain Institute, KU Leuven, Herestraat 49 bus 1023, 3000 Leuven, Belgium

2 Department of Neurobiology, Weizmann Institute of Science, Rehovot, Israel

3 Leuven Viral Vector Core, KU Leuven, Leuven, Belgium

\section{Introduction}

Mutations in leucine-rich repeat kinase 2 (LRRK2) can cause familial Parkinson's disease (PD), and variations in its locus are associated with an increased risk of developing sporadic PD [1]. Several pathogenic LRRK2 mutations increase its kinase activity $[2,3]$, and evidence from patients with sporadic PD indicates that enhanced LRRK2 kinase activity is also involved in PD pathogenesis in the absence of LRRK2 mutations [4]. Hence, pharmacological inhibition of LRRK2 kinase activity is a very attractive disease-modifying strategy [5]. Several potent and selective LRRK2 kinase inhibitors have been developed [6-8], and some are currently being tested in phase I clinical studies [9]. Nevertheless, how LRRK2 exactly contributes to the development of PD is still unclear. One of the outstanding questions is whether LRRK2 
and alpha-synuclein $(\alpha \mathrm{SYN})$ operate in the same pathological pathway. Several groups have tried to address the question whether reducing LRRK2 activity by genetic LRRK2 knock-out (KO), antisense oligonucleotides, or LRRK2 kinase inhibitors can protect against $\alpha \mathrm{SYN}$-induced neurodegeneration. These independent studies have resulted in conflicting conclusions varying from a rescue of neuronal loss [10-15], reduced pathological $\alpha \mathrm{SYN}$ levels, to a lack of neuroprotection [16-19]. Therefore, a better insight into the functional interaction between both proteins will be a key to correctly evaluate the therapeutic potential of LRRK2 inhibition.

Inflammatory pathways might provide a mechanistic link between LRRK2 and $\alpha \mathrm{SYN}$-mediated toxicity. LRRK2 kinase inhibition was shown to reduce the number of activated myeloid cells in an $\alpha \mathrm{SYN}$-based PD model [11]. Additionally, $\alpha \mathrm{SYN}$ overexpression in wild-type (WT) rats resulted in increased LRRK2 protein levels in inducible nitric oxide synthase-positive myeloid cells [11] and LRRK2 kinase inhibition was reported to increase the clearance of $\alpha \mathrm{SYN}$ by microglia [20]. Recently, transcriptome analysis of LRRK2 KO microglial cells identified alterations of inflammatory pathways upon treatment with $\alpha \mathrm{SYN}$ preformed fibrils [21]. Whether this modulatory effect on neuroinflammation is dependent on LRRK2 kinase activity is unclear, given that LRRK2 kinase inhibition did not reduce neuroinflammation in an $\alpha \mathrm{SYN}$-overexpression rat model expressing physiological LRRK2 levels [12].

Here, we aimed to further clarify the link between LRRK2 and $\alpha \mathrm{SYN}$-mediated neurodegeneration and neuroinflammation in vivo. To this end, we used a variety of recombinant adeno-associated viral (rAAV) vector-based PD rat models ranging from a modest to a more aggressive neurodegenerative phenotype. We have previously demonstrated progressive time- and dose-dependent neurodegeneration, neuropathology, and neuroinflammatory changes induced by rAAV-mediated $\alpha \mathrm{SYN}$ overexpression in the substantia nigra (SN) of the rat $[22,23]$. Here, we induced different levels of human A53T or WT $\alpha$ SYN in the SN of LRRK2 KO rats as well as WT rats with chronic pharmacological LRRK2 kinase inhibition. We found that reducing LRRK2 levels or LRRK2 kinase activity did not protect against $\alpha \mathrm{SYN}$-induced motor deficits, neurodegeneration, or $\alpha \mathrm{SYN}$ pathology regardless of the severity of the model used. Interestingly, LRRK2 $\mathrm{KO}$ rats injected with a high dose of A53T $\alpha$ SYN vector presented less reactive microglia and infiltrating immune cells compared with WT rats. Our findings show that the potentially protective effects of reduced LRRK2 activity against $\alpha \mathrm{SYN}$-induced neurodegeneration highly depend on the model used. We further identify LRRK2 as a positive regulator of ( $\alpha \mathrm{SYN}$-induced) neuroinflammation, both at the level of microglia and infiltrating $\mathrm{T}$ cells. Our results provide more insight into the physiological function of LRRK2 and reveal how its activity might be involved in pathological processes.

\section{Materials and Methods}

\section{Animals and Treatment}

All animal experiments were carried out in accordance with the European Communities Council Directive of November 24, 1986 (86/609/EEC), and approved by the Bioethical Committee of the KU Leuven (Belgium) (ECD project 2017-070). Male LRRK2 KO rats (LEH-Lrrk2 ${ }^{\text {tm } 1 \text { sage }}$ ) and WT Long-Evans controls (Horizon Discovery, Waterbeach, UK) of approximately $350 \mathrm{~g}$ were used at 8 weeks of age, unless stated differently, and housed under a normal $12 \mathrm{~h}$ light/dark cycle with free access to pelleted food and tap water. Rats were fed with food pellets containing either MLi-2 (Merck, Kenilworth, NJ) at a dose of $15 \mathrm{mg} / \mathrm{day} / \mathrm{kg}$ of body weight (Research Diets, Inc., New Brunswick, NJ) or placebo starting on day 4 after injection and maintained for the duration of the experiment. Animals were randomly assigned to the compound or placebo groups. For biochemical analysis of tyrosine hydroxylase (TH), acute LRRK2 kinase inhibition was obtained via intraperitoneal (i.p.) injection of MLi-2 $(10 \mathrm{mg} / \mathrm{kg})$ in $20 \% \beta$-cyclodextrin or vehicle alone twice a day during the last 5 days of the experiment.

\section{Recombinant AAV Production and Purification}

Vector production and purification were performed as previously described [24]. The plasmids include the constructs for the AAV7 serotype, the AAV transfer plasmid encoding human A53T mutant or WT $\alpha$ SYN under the control of the ubiquitous CMVie-enhanced synapsin 1 promoter, and the pAdvDeltaF6 adenoviral helper plasmid. Real-time PCR analysis was used for genomic copy determination.

\section{Stereotactic Vector Injections}

All surgical procedures were performed using aseptic techniques and ketamine $(60 \mathrm{mg} / \mathrm{kg}$, i.p., Ketalar, Pfizer, Puurs, Belgium) and medetomidine $(0.4 \mathrm{mg} / \mathrm{kg}$, Dormitor, Pfizer $)$ anesthesia. Following anesthesia, the rodents were placed in a stereotactic head frame (Stoelting, Wood Dale, IL). Injections were performed with a 30 -gauge needle and a 10- $\mu$ l Hamilton syringe (Hamilton, Bonaduz, GR, Switzerland). LRRK2 KO or WT rats were injected with $3 \mu 1 \mathrm{rAAV} 2 / 7 \mathrm{~A} 53 \mathrm{~T}$ or WT $\alpha \mathrm{SYN}$ vector (low vector dose: $3.0 \mathrm{E}+11$ genome copies $(\mathrm{GC}) / \mathrm{ml}$, medium vector dose: $4.0 \mathrm{E}+11 \mathrm{GC} / \mathrm{ml}$, high vector dose: $9.0 \mathrm{E}+11 \mathrm{GC} / \mathrm{ml})$. An additional group of rats was injected with a high dose $(9.0 \mathrm{E}+$ 
$11 \mathrm{GC} / \mathrm{ml}$ ) rAAV2/7 eGFP control vector. Stereotactic coordinates used for the SN were anteroposterior $(-5.3)$, lateral $(-2.0)$, and dorsoventral ( -7.2$)$, calculated from the dura using bregma as reference. The injection rate was $0.25 \mu \mathrm{l} / \mathrm{min}$, and the needle was left in place for an additional $5 \mathrm{~min}$ period before being retracted.

\section{Cylinder Test}

The cylinder test was employed to quantify forelimb use. Contacts made by each forepaw with the wall of a $20-\mathrm{cm}-$ wide clear glass cylinder were scored from the videotapes by an observer blinded to the animal's identity. A total of 25 contacts were recorded for each animal. The number of contralateral forelimb contacts was expressed as a percentage of total forelimb contacts. Nonlesioned control rats score around $50 \%$ in this test.

\section{Immunohistochemistry}

Rats were sacrificed with an overdose of sodium pentobarbital (200 mg/kg, i.p., Dolethal, Vetoquinol, Lure, France) followed by 2-step intracardial perfusion with saline and $4 \%$ paraformaldehyde (PFA) in PBS. After post-fixation overnight in 4\% PFA, 50 - $\mu \mathrm{m}$-thick coronal brain sections were made with a vibrating microtome (HM 650V, Microm, Walldorf, Germany). Immunostainings were performed on free-floating sections using antibodies against TH (AB152 rabbit polyclonal 1:1000, Millipore, Overijse, Belgium), $\alpha \mathrm{SYN}$ (AB 5038 rabbit polyclonal 1:5000, Millipore), phosphorylated $\alpha \mathrm{SYN}(\mathrm{P}-\alpha \mathrm{SYN}$ mouse 11A5, 1:5000, provided by Elan Pharmaceuticals, Inc., Dublin, Ireland), Iba1 (goat polyclonal 1:1000, Abcam, Cambridge, UK), and MHC II (mouse MCA46G 1:250, Serotec, Temse, Belgium). For chromogen-based immunohistochemistry (TH, $\alpha \mathrm{SYN}$, Iba1, and MHC II), sections were pretreated with 3\% hydrogen peroxide for $10 \mathrm{~min}$ and incubated with $10 \mathrm{mM}$ sodium citrate $(\mathrm{pH} 6.0)$ at $80{ }^{\circ} \mathrm{C}$ for $20 \mathrm{~min}$, followed by incubation on ice for $10 \mathrm{~min}$. Sections were treated with $10 \%$ normal serum (matched to secondary antibody, DakoCytomation, Leuven, Belgium) in $0.1 \%$ Triton X-100 in PBS and incubated overnight with primary antibody. As secondary antibody, biotinylated antirabbit IgG (1:600 for TH and $\alpha \mathrm{SYN}$, DakoCytomation), antigoat IgG (1:300 for Iba1, DakoCytomation), and anti-mouse IgG (1:500 for MHCII, DakoCytomation) were used, followed by incubation with streptavidin-horseradish peroxidase complex (1:1000, DakoCytomation). TH immunoreactivity was visualized using Vector SG (SK-4700, Vector Laboratories, Burlingame, CA), and $\alpha \mathrm{SYN}$ and Ibal immunoreactivity was visualized using 3,3-diaminobenzidine $(0.4 \mathrm{mg} / \mathrm{ml}$, SigmaAldrich, St. Louis, MS) as a chromogen. For immunofluorescence staining (P- $\alpha \mathrm{SYN}$ and $\mathrm{TH})$, sections were rinsed three times in PBS and then incubated overnight in PBS- $0.1 \%$ Triton X-100 with $10 \%$ donkey serum (Jackson
ImmunoResearch Laboratories, Inc., Ely, UK) and with the primary antibody. After three rinses in PBS- $0.1 \%$ Triton X-100, the sections were incubated in the dark for $2 \mathrm{~h}$ in fluorochromeconjugated secondary antibodies: donkey anti-rabbit Alexa 488 (1:1000, Molecular Probes, Invitrogen, Merelbeke, Belgium) and donkey anti-mouse Cy3 (1:500, Jackson ImmunoResearch, Uden, The Netherlands). After being rinsed in PBS and mounted, the sections were coverslipped with Mowiol 488 (Calbiochem, San Diego, CA) and DAPI (1:1000).

\section{Stereological Quantification of Dopaminergic Neurons}

The number of $\mathrm{TH}^{+}$cells in the $\mathrm{SN}$ was determined by stereological measurements using the optical fractionator method in a computerized system as described before [25] (Stereo Investigator; MicroBrightField (MBF), Delft, The Netherlands). Every fifth section throughout the entire SN was analyzed, with a total of 7 sections for each animal. The coefficients of error, calculated according to the procedure of Schmitz and Hof [26] as estimates of precision, varied between 0.05 and 0.10 . Percentages represent the loss of dopaminergic neurons compared to the contralateral, non-injected side. For the CD68 and Ibal stereological quantifications, we made use of the software Stereologer, SRC Biosciences (Stereology Resource Center, Saint Petersburg, FL). Microglial morphology was assessed using the IUR Nucleator (volume) and IUR-Line (length) probes. All analyses were performed by an investigator blinded to the different groups.

\section{Quantification of Striatal Dopaminergic Terminals}

Seven sections covering the whole striatum were stained against $\mathrm{TH}$ as previously described. Images were acquired using the Leica DM4 B optical microscope (Leica, Wetzlar, Germany) with a Leica DFC 320 digital camera (Leica) and the Leica Application Suite software (Leica). Intensity measurement was performed using the software ImageJ. Percentages represent the loss of striatal TH compared to the contralateral, non-injected side.

\section{Western Blot Analysis}

Nigral and striatal tissues were freshly isolated and homogenized in 5 volumes of buffer $(10 \mathrm{mM}$ Tris- $\mathrm{HCl}, 10 \mathrm{mM}$ EDTA, $0.25 \mathrm{mM}$ sucrose, $\mathrm{pH}$ 7.5) containing a protease inhibitor cocktail (Roche cOmplete) and phospho-STOP EASYPACK (Roche, Anderlecht Belgium) using a Dounce tissue grinder followed by sonication (15 s, duty cycle $30 \%$, output 2 using the Branson Sonifier 250). The brain samples used to determine $\alpha \mathrm{SYN}$ expression levels were additionally spun for $10 \mathrm{~min}$ at $6000 \mathrm{~g}$ at $4{ }^{\circ} \mathrm{C}$, and the resulting supernatants represented the protein fraction. Kidney tissue was 
homogenized in 10 volumes of urea buffer $(8 \mathrm{M}$ urea, $100 \mathrm{mM}$ Tris- $\mathrm{HCl}, 10 \%$ glycerol, $1 \%$ SDS, 5 mM DTT, $1 \mathrm{mM}$ EDTA, $1 \mathrm{mM}$ EGTA, $\mathrm{pH}$ 6.8) containing protease and phosphatase inhibitor using a Dounce tissue grinder. Protein sample concentration was determined by BCA protein assay (Thermo Scientific, MA, USA) according to the manufacturer's directions.

To assess LRRK2, LRRK2 P-S935, and TH levels by Western blot analysis, $40 \mu \mathrm{g}$ protein was mixed with a $6 \times$ denaturing loading buffer $(50 \mathrm{mM}$ Tris- $\mathrm{HCl}, \mathrm{pH} 6.8,4 \%$ SDS, $10 \% \beta$-mercaptoethanol, $12 \%$ glycerol, and $0.01 \%$ bromophenol blue). Samples were heated to $95{ }^{\circ} \mathrm{C}$ for $10 \mathrm{~min}$ and ran on NuPAGE 3-8\% Tris-acetate gradient gels (Invitrogen, Waltham, MA). Separated proteins were transferred to a polyvinylidene fluoride (PVDF) membrane (BioRad, Irvine, CA). Nonspecific binding sites were blocked for 15 min in PBS with $0.1 \%$ Triton X-100 (PBS-T) and 5\% nonfat milk. After overnight incubation at $4{ }^{\circ} \mathrm{C}$ with primary antibodies (anti-LRRK2 N241A/34 1:200, NeuroMab, CA; anti-LRRK2 (P-S935) 1:100, Abcam; anti-TH Ab152 1:1000, Millipore; anti-vinculin V9131 1:100,000, Sigma; anti- $\alpha$ Tubulin T5168 1:10,000, Sigma), blots were washed 3 times with PBS-T and incubated with horseradish peroxidaseconjugated secondary antibody (Dako, Glostrup, Denmark) for $1 \mathrm{~h}$ and washed again 3 times.

To measure the $\alpha \mathrm{SYN}$ expression levels in the nigral brain samples, $15 \mu \mathrm{g}$ of protein was mixed with a $6 \times$ loading buffer, boiled for $10 \mathrm{~min}$, and separated on a 4-15\% Tris-glycine (Bio-Rad) gel with Novex Tris-glycine-SDS running buffer and the PageRuler Plus molecular weight marker. After electrophoresis, proteins were blotted onto a PVDF membrane (Bio-Rad) for $30 \mathrm{~min}$ (Trans-Blot Turbo Transfer system) in TurboBlotter transfer buffer. After transfer, membranes were incubated with $0.4 \%$ paraformaldehyde for $20 \mathrm{~min}$ at RT, rinsed $3 \times$ with PBS-T, and blocked in 5\% nonfat milk in PBS-T for 30-60 min. After blocking, membranes were incubated with rabbit anti- $\alpha$ SYN primary antibody (in-house, $1: 2000$ ) in $5 \%$ milk in PBS-T overnight at $4{ }^{\circ} \mathrm{C}$. Anti- $\beta$ tubulin (Sigma T4026, 1:10,000) was used as internal loading control. After rinsing $3 \times$ with PBS-T, blots were incubated with horseradish peroxidase-conjugated secondary antibodies. Bands were visualized using Clarity Western ECL (BioRad) and developed with a GE ImageQuant 800 (GE Healthcare, Bloomington, IL). Densitometric analysis was performed using ImageQuant software (GE Healthcare).

\section{Flow Cytometry}

Two-year-old LRRK2 KO and WT control male rats were bilaterally injected in the SN with $3 \mu \mathrm{l}$ of rAAV2/7 A53T $\alpha \mathrm{SYN}$ vector (high vector dose: $9.0 \mathrm{E}+11 \mathrm{GC} / \mathrm{ml}$ ). Two weeks post injection, the animals were sacrificed with an overdose of sodium pentobarbital (Dolethal, $200 \mathrm{mg} / \mathrm{kg}$, i.p.), followed by intracardial perfusion with ice-cold saline. Midbrains were dissected and mechanically dissociated into a single-cell suspension with a Dounce homogenizer, followed by passage through a 40- $\mu \mathrm{m}$ cell strainer. Cells were washed with Hank's Balanced Salt Solution (HBSS) and centrifuged at $400 \mathrm{~g}$ for $3 \mathrm{~min}$. The pellet was resuspended in a $37 \%$ Percoll (GE Healthcare BioSciences) gradient and centrifuged for $25 \mathrm{~min}$ at $800 \mathrm{~g}$ with slow acceleration and no brake. Cells were collected from the bottom of the tube and washed twice with DMEM containing $10 \%$ fetal bovine serum (FBS) and once with FBS stain buffer (BD Biosciences, Franklin Lakes, NJ). Cells were blocked with mouse anti-rat Fc $\gamma$ II receptor (CD32) (1:500, D34-485, BD Biosciences) and stained for the following surface markers: CD45 OX1, FITC (eBioscience, Affymetrix, San Diego, CA); MHC II HIS19, APC (eBioscience); CD3 eBioG4.18, PE (eBioscience); CD8a OX8, PE-Cyanine7 (eBioscience); CD11b WT.5 (RUO), BV450 (BD Biosciences); and CD4 OX-35 (RUO), BV510 (BD Biosciences). A fixable viability dye eFluor 780 (eBioscience) was used in each condition. Cells were fixed in $4 \%$ PFA, and data was acquired on the following day. Fluorescence Minus One (FMO) controls of each marker were included. Flow cytometry/FACS was performed at the VIB-KU Leuven FACS Core Facility using a BD FACSCanto II cytometer with the FACSDiva software. Data was analyzed using FlowJo v10.0.7 software. Fifty thousand events were collected for each brain sample.

\section{Statistical Analyses}

Graph creation and statistical analysis were performed using GraphPad Prism for Windows (GraphPad Software, Inc.), version 8.0. Results are presented as means \pm standard error of the mean. Normality of data was tested using the Shapiro-Wilk test. Statistical significance was assessed using either a $t$ test or when multiple groups were analyzed simultaneously, either one-way ANOVA followed by Tukey's multiple comparison post hoc test or two-way ANOVA followed by Sidak's post hoc test. For non-normal distributed data, statistical significance was assessed using the Kruskal-Wallis test followed by Dunn's multiple comparison post hoc test. Significance was represented as follows: $* p<0.05, * * p<0.01$, $* * * p<0.001$, and $* * * * p<0.0001$.

\section{Results}

\section{LRRK2 Ablation Does Not Affect aSYN-Induced Progressive Motor Deficits In Vivo}

To explore the functional interaction between LRRK2 and $\alpha \mathrm{SYN}$ and to evaluate LRRK2 activity as a therapeutic target, we studied the effects of reduced LRRK2 activity on different aspects of $\alpha \mathrm{SYN}$-mediated neurodegeneration and 
neuroinflammation. To this end, we applied our in-house-developed rat PD model, based on rAAV-mediated overexpression of human $\alpha \mathrm{SYN}$ (rAAV2/7 $\alpha \mathrm{SYN}$ ) in the dopaminergic neurons of the SN [23]. We have previously extensively characterized this model and showed that the phenotypes investigated in this study (i.e., nigral dopaminergic neurodegeneration, striatal TH loss, motor behavior deficits, and sustained neuroinflammation) are specific for $\alpha \mathrm{SYN}$, as none of these phenotypes could be detected in control conditions injected with a rAAV-GFP vector $[23,27]$. Here, A53T or WT $\alpha$ SYNencoding vectors were injected in WT and LRRK2 KO rats (Fig. 1A). The lack of LRRK2 protein in the LRRK2 KO rats was confirmed via Western blotting (Fig. S1C). By applying different vector doses (low: 3E11 GC/ml, medium: 4E11 GC/ $\mathrm{ml}$, and high: $9 \mathrm{E} 11 \mathrm{GC} / \mathrm{ml}$ ), we induced different levels of $\alpha \mathrm{SYN}$ overexpression (Fig. S2) to obtain distinct levels of neurodegeneration ranging from a modest to a more severe phenotype. To monitor motor performance, we subjected the
Fig. 1 LRRK2 ablation does not protect against $\alpha \mathrm{SYN}$-induced progressive motor deficits in vivo. (A) Schematic representation of the experimental setup. WT $(+/+)$ and LRRK2 $\mathrm{KO}(-/-)$ rats were injected with different doses of rAAV2/7 vectors encoding A53T or WT $\alpha \mathrm{SYN}$ in the SN. Behavioral tests were performed 2 weeks and 4 weeks post injection (p.i.). In addition, a group of WT rats was treated daily with $\mathrm{MLi}-2$ or placebo via food pellets from day 4 to 28 . All animals were sacrificed 4 weeks p.i., and the brains were processed for histological analysis. (B, C) Left forepaw use in the cylinder test for WT and LRRK2 KO rats 2 weeks and 4 weeks p.i. with rAAV2/7 A53T (low, medium, and high doses) (B) or WT $\alpha \mathrm{SYN}$ vector (low and medium doses) (C). Graphs represent mean \pm S.E.M. Statistical differences were assessed using two-way ANOVA and Sidak's post hoc test, $n=8-11$ animals/group. WT versus LRRK2 KO: A53T $\alpha \mathrm{SYN}, F(5,107)=1.933$; WT $\alpha \mathrm{SYN}, F(3,72)=1.200 ; * p=$ 0.0251 . Vector dose effect: A53T $\alpha \mathrm{SYN}, F(2,50)=1.864$, $p>0.05$; WT $\alpha \mathrm{SYN}, F(1,36)=$ $0.1768, p>0.05$
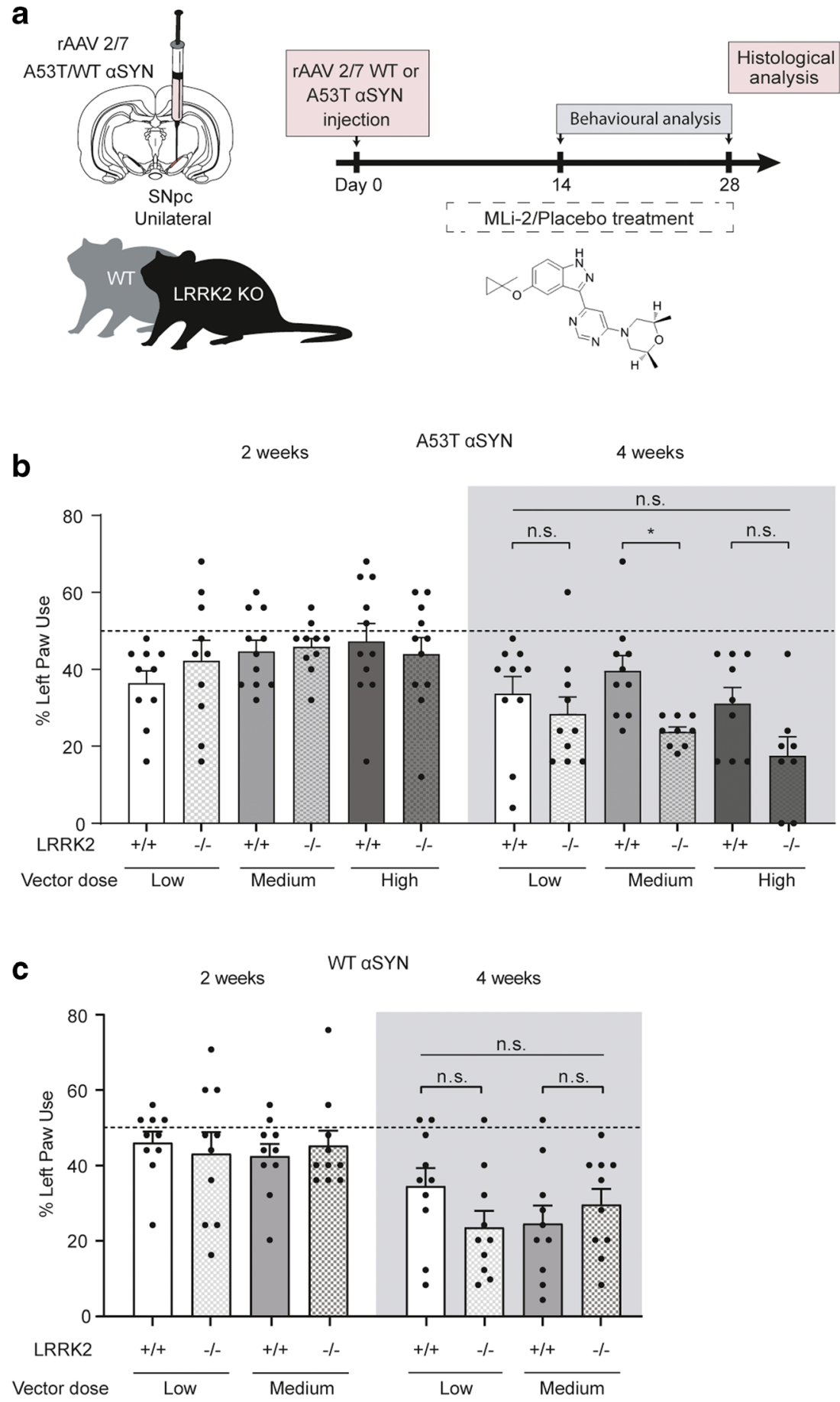
A53T and WT $\alpha$ SYN-injected animals to a cylinder test (Fig. 1B, C), which revealed progressive motor deficits over time. As a control, we injected a high dose $(9 \mathrm{E} 11 \mathrm{GC} / \mathrm{ml})$ of an eGFP vector in WT animals and confirmed the lack of behavioral deficits (Fig. S3A). Loss of LRRK2 did not attenuate the $\alpha \mathrm{SYN}$-induced motor deficits. On the contrary, all the A53T $\alpha \mathrm{SYN}-$ injected KO animals displayed a trend towards increased motor deficits, which was significant in the medium-vector dose group (Fig. 1B). We can conclude that under our experimental conditions, LRRK2 deletion did not abate the $\alpha \mathrm{SYN}$-induced motor deficits in mild or severe A53T or WT $\alpha \mathrm{SYN}$-based PD models. In some of the conditions, it even worsened the motor behavior.

\section{Reduced LRRK2 Activity Does Not Attenuate aSYN- Induced Neurodegeneration In Vivo}

Next, we assessed the level of nigral dopaminergic neurodegeneration induced by overexpression of different levels of A53T or WT $\alpha$ SYN. We observed loss of the dopaminergic neurons in the SN of all the rAAV2/7 $\alpha \mathrm{SYN}$-injected animals 4 weeks post injection (p.i.) (Fig. 2A-C), whereas no neurotoxicity was observed in WT animals injected with a high dose of eGFP vector (Fig. S3B). Injection with a low, medium, or high vector dose of $\mathrm{AAAV} 2 / 7 \mathrm{~A} 53 \mathrm{~T} \alpha \mathrm{SYN}$ resulted in a dosedependent $\mathrm{TH}^{+}$neuronal cell loss of $33 \%, 43 \%$, and $60 \%$, respectively. Injection with a low or a medium vector dose of rAAV2/7 WT $\alpha \mathrm{SYN}$ resulted in a lesion size of $45 \%$ and $59 \%$, respectively. However, no significant differences were observed between WT and LRRK2 KO animals for both the rAAV2/7 A53T- and WT $\alpha \mathrm{SYN}$-injected animals at any vector dose. Quantification of the dopaminergic nerve terminals revealed a significant striatal lesion upon injection of different doses of A53T (ranging from 35 to $85 \%$ ) or WT (ranging from 40 to $70 \%$ ) rAAV2/7 $\alpha \mathrm{SYN} 4$ weeks p.i. In line with the dopaminergic cell loss, no significant differences were detected between WT and LRRK2 KO animals in all the different conditions evaluated (Fig. 2E-G).

Using a similar experimental setup, we evaluated the effect of pharmacological LRRK2 kinase inhibition. rAAV2/7 WT $\alpha \mathrm{SYN}$-injected WT rats (medium vector dose) were treated with MLi-2, one of the most potent LRRK2 kinase inhibitors currently available, or placebo. The LRRK2 kinase inhibitor was administered at a concentration of $15 \mathrm{mg} / \mathrm{kg} /$ day for 4 weeks using an in-diet treatment protocol [6] with an actual mean intake of $13 \mathrm{mg} / \mathrm{kg} /$ day. The treatment was well tolerated without differences in animal weight between the treatment and placebo groups (Fig. S1A). Inhibition of LRRK2 kinase activity in the brain induced by chronic MLi-2 in-diet treatment was reported previously $[6,28]$ and observed in-house in mice (data not shown). Here, we confirmed MLi-2-induced inhibition of LRRK2 kinase activity by showing dephosphorylation at LRRK2 S935 in the kidney of the same animals used to study $\alpha \mathrm{SYN}$-induced effects in the brain (Fig. S1B). Treatment with MLi- 2 did not alter $\alpha$ SYN-mediated nigral dopaminergic cell loss (Fig. 2D). Similarly, no difference in striatal lesion was found in WT animals treated with the MLi2 compound compared to placebo (Fig. $2 \mathrm{H}$ ).

\section{Reduced LRRK2 Activity Alters Nigral and Striatal TH Levels}

Although no significant differences in neurotoxicity were detected, we observed a trend towards increased nigral cell loss in the LRRK2 KO animals injected with a medium and high rAAV2/7 A53T $\alpha$ SYN vector dose (Fig. 2B, $\mathrm{F}$ ), in line with the observed motor deficits (Fig. 1B). To investigate this unexpected finding more thoroughly, we used immunoblotting to assess the levels of $\mathrm{TH}$ in nigral and striatal brain fractions of WT and LRRK2 KO animals injected with a medium dose of rAAV2/7 A53T $\alpha \mathrm{SYN}$. Densitometry analysis revealed significantly lower TH levels in the SN and the striatum of LRRK2 KO animals compared with those of WT rats (Fig. 2I, J). In addition, we treated a subgroup of $\alpha \mathrm{SYN}$-injected WT animals with MLi-2 via i.p. injections $(10 \mathrm{mg} / \mathrm{kg}$ for 5 consecutive days), inducing acute inhibition of LRRK2 kinase activity (Fig. S1C). Western blot analysis revealed a significant decrease in TH levels in the striatum but not in the SN of MLi-2-treated rats compared with placebotreated animals (Fig. 2I, J). Together, these results suggest that both loss of LRRK2 and reduced LRRK2 kinase activity affect $\mathrm{TH}$ levels in $\alpha \mathrm{SYN}$-overexpressing nigral neurons.

\section{Reduced LRRK2 Activity Does Not Affect aSYN Pathology}

Next, we assessed the effect of genetic LRRK2 ablation and pharmacological LRRK2 kinase inhibition on the phosphorylation of $\alpha$ SYN. Previous studies have shown that $\alpha \mathrm{SYN}$ phosphorylated at serine 129 consistently accumulates in Lewy bodies [29]. Therefore, the presence of phosphorylated $\alpha \mathrm{SYN}$ in the dopaminergic neurons of the $\mathrm{SN}$ is considered a pathological marker of PD. Immunofluorescent double stainings for $\mathrm{P}-\alpha \mathrm{SYN}$ and $\mathrm{TH}$ revealed clear $\mathrm{P}-\alpha \mathrm{SYN}-$ positive nigral neurons 4 weeks p.i. in all the experimental conditions (Figs. 3A and S4). However, no significant difference in the number of P- $\alpha \mathrm{SYN}$-positive dopaminergic neurons was observed between WT and LRRK2 KO animals at any dose of rAAV2/7 A53T or WT $\alpha$ SYN (Fig. 3B, C). Similarly, no difference in the number of $\mathrm{P}-\alpha \mathrm{SYN}$-positive dopaminergic neurons was observed between the MLi-2-treated and placebo-treated animals (Fig. 3D). 
a

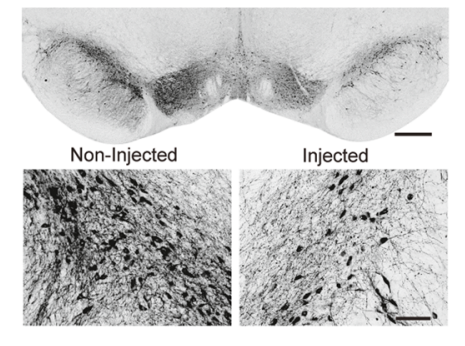

e

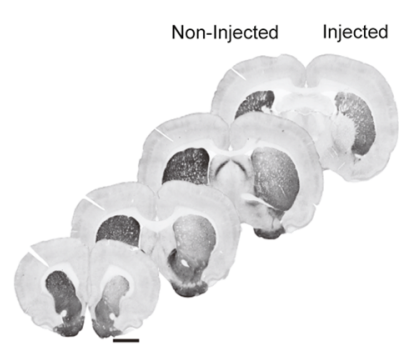

i

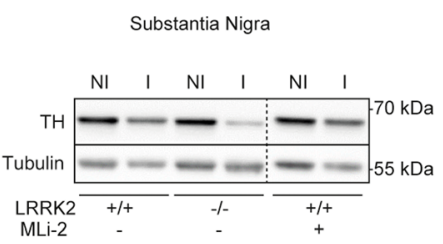

Fig. 2 Reduced LRRK2 activity does not attenuate $\alpha \mathrm{SYN}$-induced neurotoxicity in vivo but alters TH levels. (A) Representative image of TH immunostaining in the SN 4 weeks p.i. of a medium-dose rAAV2/7 WT $\alpha \mathrm{SYN}$ in LRRK2 KO rats. Scale bars $=500 \mu \mathrm{m}$ (top) and $50 \mu \mathrm{m}$ (bottom). (B-D) Stereological quantification of the nigral lesion in the injected SN relative to the non-injected side 4 weeks p.i. in WT and LRRK2 KO rats injected with different doses of rAAV2/7 A53T $\alpha$ SYN (mean \pm S.E.M., two-way ANOVA and Sidak's post hoc test, $n=8-10$ animals/group. WT versus LRRK2 KO: $F(1,45)=1.607, p>0.05$.

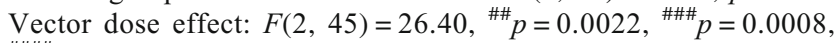
\#\#\#\# $p<0.0001$ (B), rAAV2/7 WT $\alpha \mathrm{SYN}$ (mean \pm S.E.M., two-way ANOVA and Sidak's post hoc test, $n=7-9$ animals/group; WT versus LRRK2 KO: $F(1,29)=2.894, p>0.05$; vector dose effect: $F(1,29)=$ $\left.7.183,{ }^{\#} p=0.012\right)(\mathrm{C})$ or rAAV2/7 WT $\alpha \mathrm{SYN}-$ injected WT rats treated with MLi-2 or placebo (mean \pm S.E.M., $t$ test, $n=6-7$ animals/group, $F(7,5)=1.155, p>0.05$ ) (D). (E) Representative image of immunostaining for TH in the striatum after injection of a medium-dose rAAV2/7 WT $\alpha \mathrm{SYN}$ in LRRK2 KO rats. Scale bar $=2 \mathrm{~mm}$. $(\mathrm{F}-\mathrm{H})$ Quantification of TH immunolabeling in striatal dopaminergic terminals in the injected relative

\section{Loss of LRRK2 Attenuates aSYN-Induced Microglial Activation and T Cell Infiltration}

Given the role of both LRRK2 and $\alpha \mathrm{SYN}$ in neuroinflammatory processes, we investigated whether
C

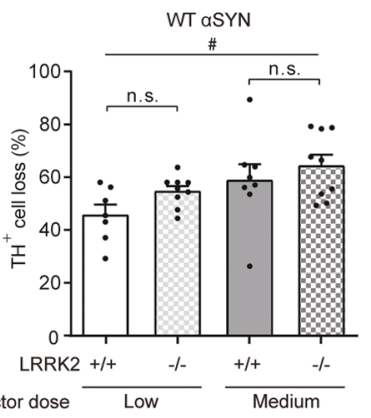

d WT aSYN

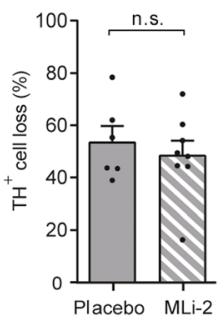

g WT aSYN

h WT aSYN
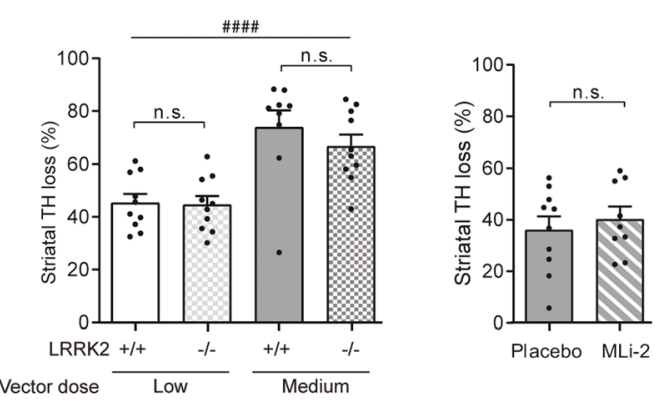

j
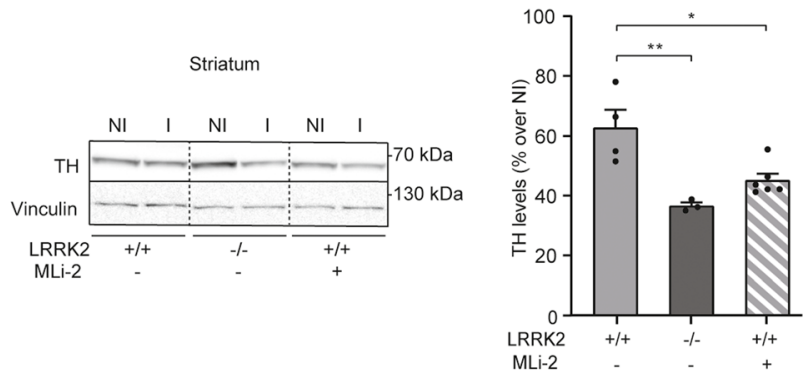

to the non-injected side 4 weeks p.i. in WT and LRRK2 KO rats after different doses of rAAV2/7 A53T $\alpha$ SYN (mean \pm S.E.M., two-way ANOVA and Sidak's post hoc test, $n=9-11$ animals/group; WT versus LRRK2 KO: $F(1,54)=4.774, p>0.05$; vector dose effect: $F(2,54)=$ $41.74, \ldots+0.0001)(\mathrm{F}), \mathrm{rAAV} 2 / 7 \mathrm{WT} \alpha \mathrm{SYN}$ (mean \pm S.E.M., twoway ANOVA and Sidak's post hoc test, $n=9-10$ animals/group; WT versus LRRK2 KO: $F(1,36)=0.9824, p>0.05$; vector dose effect: $F(1$, $36)=35.53, \ldots+0.0001)(\mathrm{G})$, or rAAV2/7 WT $\alpha \mathrm{SYN}$-injected WT rats treated with MLi- 2 or placebo (mean \pm S.E.M., $t$ test, $n=9$ animals/ group, $F(9,8)=1.384, p>0.05)(\mathrm{H})$. (I, J) Immunoblot analysis of TH levels in nigral (I) and striatal tissue (J) of LRRK2 KO and WT rats injected with a medium-dose rAAV2/7 A53T $\alpha \mathrm{SYN}$-treated with MLi2 or placebo. NI: non-injected, I: injected. Quantitative analyses were based on densitometry and present TH levels relative to vinculin or $\alpha$ tubulin levels. TH levels are expressed as a percentage of ipsilateral/ contralateral side (mean \pm S.E.M., one-way ANOVA and Tukey's post hoc test, $n=3-6$ animals/group, $F(2,9)=1.290, * * p=0.007$ (I); $F(2$, $10)=3.477,{ }^{*} p=0.013$ and $\left.{ }^{* *} p=0.003(\mathrm{~J})\right)$

reducing LRRK2 activity affects the $\alpha \mathrm{SYN}$-induced inflammatory response. First, we quantified the number of Iba1positive microglia in the SN (Fig. 4A-D). Overexpression of $\alpha \mathrm{SYN}$ increased the number of Iba1-positive cells, as previously described [27]. However, no significant difference was 
a

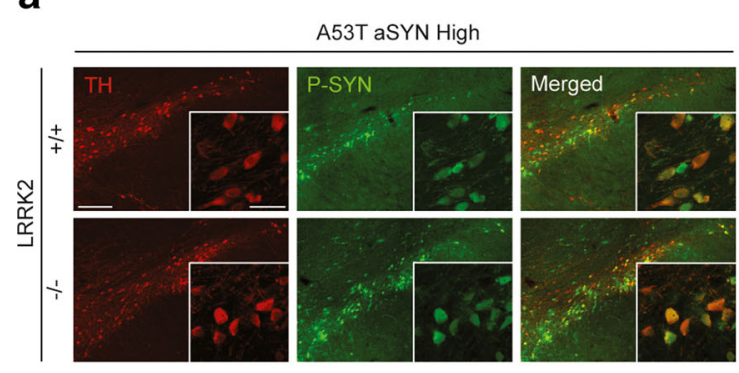

C

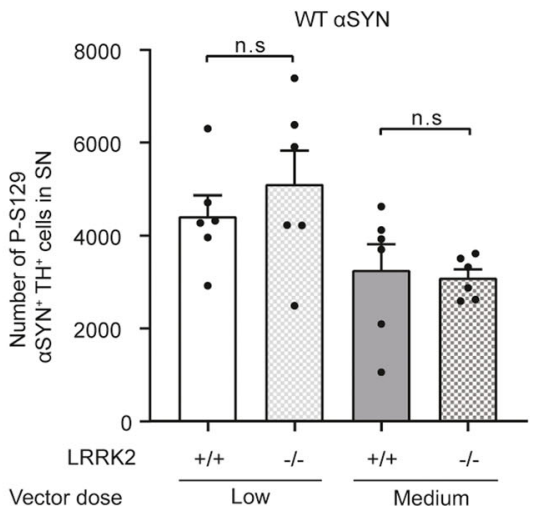

Fig. 3 Reduced LRRK2 activity does not affect $\alpha$ SYN pathology. (A) Representative image of a double immunostaining for TH and pSer129$\alpha \mathrm{SYN}$ after injection of a high dose of rAAV2/7 A53T $\alpha \mathrm{SYN}$ vector in the SN of LRRK2 KO rats. Scale bars $=250 \mu \mathrm{m}$ (main picture) or $25 \mu \mathrm{m}$ (inset). (B, C) Stereological quantification of the number of P- $\alpha \mathrm{SYN}^{+}$ $\mathrm{TH}^{+}$cells in the SN of WT and LRRK2 KO rats injected with different doses of rAAV2/7 A53T $\alpha$ SYN (low, medium, high) (B) or rAAV2/7

detected between WT and LRRK2 KO animals for the different doses of rAAV2/7 A53T (Fig. 4A, B) or WT $\alpha \mathrm{SYN}$ (Fig. 4A, C). Also, pharmacological LRRK2 kinase inhibition did not affect the number of Iba1-positive cells in the SN (Fig. 4A, D). Under inflammatory conditions, microglia shift from a highly ramified to an ameboid conformation with increased soma size and decreased length of the processes. Therefore, we also performed a stereological analysis of the microglial morphology (Fig. S5, Fig. 4A, E-G). Interestingly, significant morphological differences were observed in the SN of LRRK2 KO and WT animals injected with a high dose of rAAV2/7 A53T $\alpha$ SYN vector. Microglia of LRRK2 KO animals displayed more processes and a smaller soma size compared with microglia of WT animals, suggesting a role for LRRK2 in the activation of these cells upon high levels of A53T $\alpha$ SYN overexpression (Fig. 4E). Next, we assessed expression of major histocompatibility complex class II (MHC II) in the SN as a marker for reactive antigenpresenting cells in LRRK2 KO and WT rats injected with the different doses of $\mathrm{rAAV} 2 / 7 \mathrm{~A} 53 \mathrm{~T}$ or WT $\alpha \mathrm{SYN}$ vector (Fig. 5A-D). A significant reduction in the number of MHC
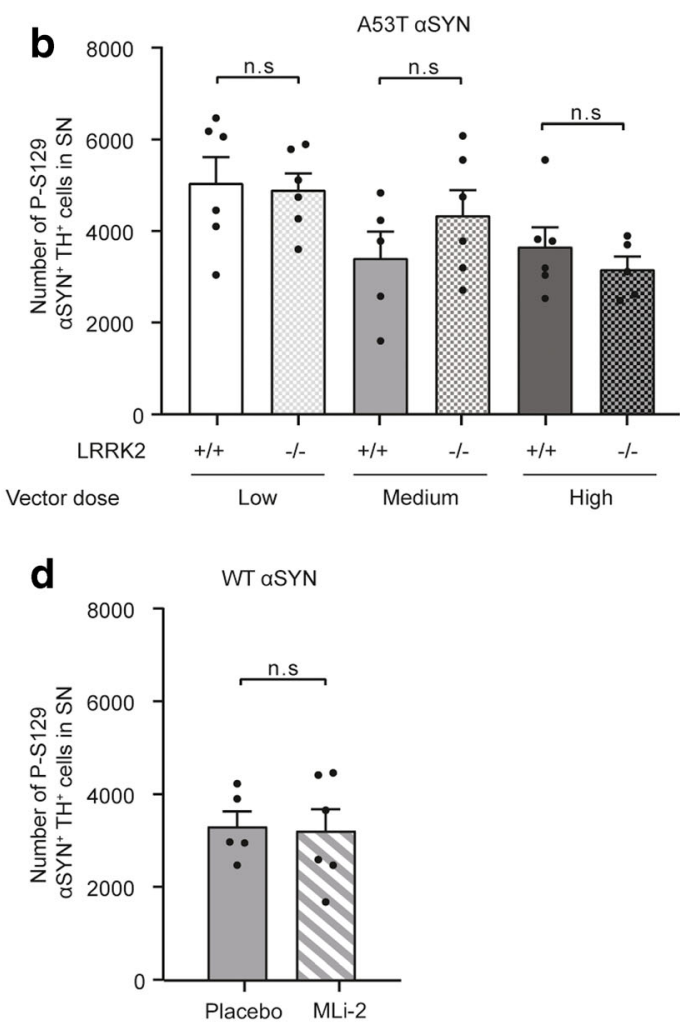

WT $\alpha$ SYN (low, medium) vector (C) (mean \pm S.E.M., two-way ANOVA and Sidak's post hoc test, $n=6$ animals/group, $F(5,28)=2.711, p>0.05$ (B); $F(3,20)=3.411, p>0.05$ (C)). (D) Stereological quantification of the number of $\mathrm{P}-\alpha \mathrm{SYN}^{+} \mathrm{TH}^{+}$cells in $\mathrm{SN}$ of WT rats injected with rAAV2/7 WT $\alpha$ SYN (medium dose) and treated with MLi- 2 or placebo (mean \pm S.E.M., $t$ test, $n=5-6, F(5,4)=2.423, p>0.05$ )

II-positive cells was observed in LRRK2 KO animals compared with the WT animals injected with the high dose of A53T, as well as both low and medium doses of WT $\alpha$ SYN vector. No significant differences were observed in animals treated with $\mathrm{MLi}-2$. Of note, WT $\alpha \mathrm{SYN}$ overexpression induced a significantly stronger MHCII response compared to the mutant A53T protein (Fig. S6).

Considering the significant differences in microglial morphology and MHC II expression (Figs. 4E and 5B), we performed flow cytometric immunophenotyping on the midbrain of LRRK2 $\mathrm{KO}$ and WT animals injected with a high dose of rAAV2/7 A53T $\alpha$ SYN vector (Fig. 5E-I). We analyzed the expression level of MHC II in microglia to assess their activation state, as well as the frequency of infiltrating $\mathrm{CD}^{+}$and $\mathrm{CD} 8^{+} \mathrm{T}$ cells (gating strategy is shown in Fig. 5E). In line with the stereological quantifications, we found a significant reduction in the expression of MHC II in microglia from LRRK2 KO rats compared to WT animals (Fig. 5F, G). In addition, the frequency of $\mathrm{CD}^{+}{ }^{+}$and $\mathrm{CD} 8^{+} \mathrm{T}$ cells infiltrating the brain was significantly lower in LRRK2 KO animals (Fig. 5H, I). 
a
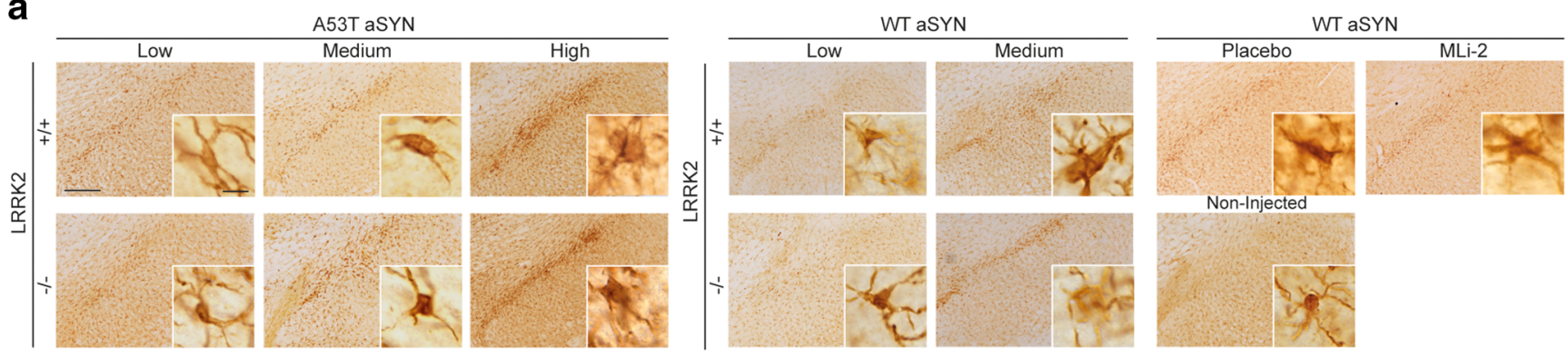

b

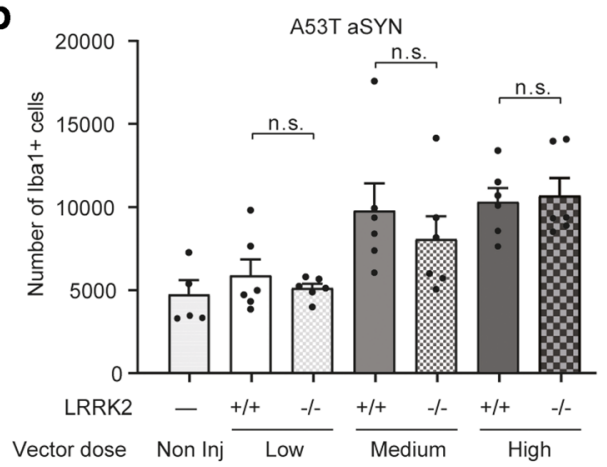

e

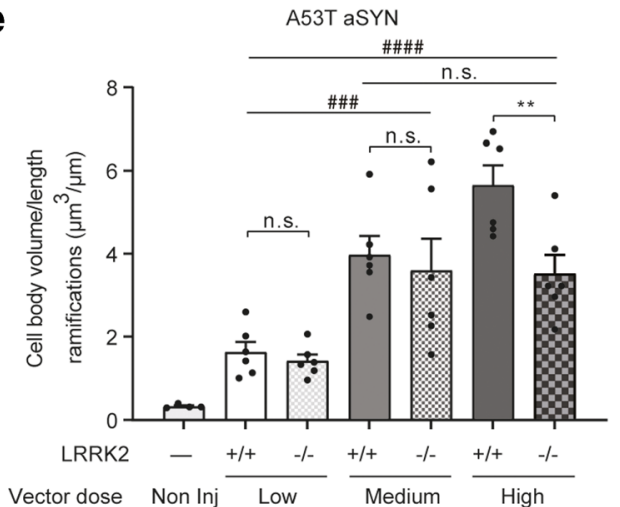

C

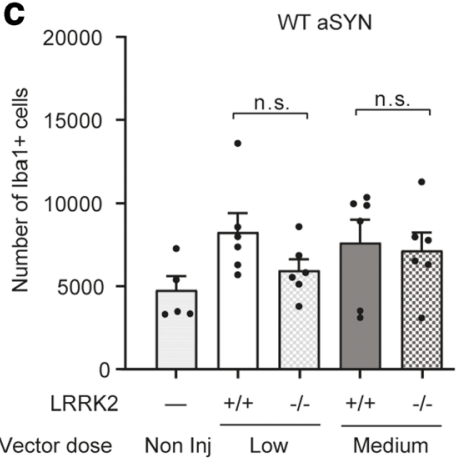

$\mathbf{f}$

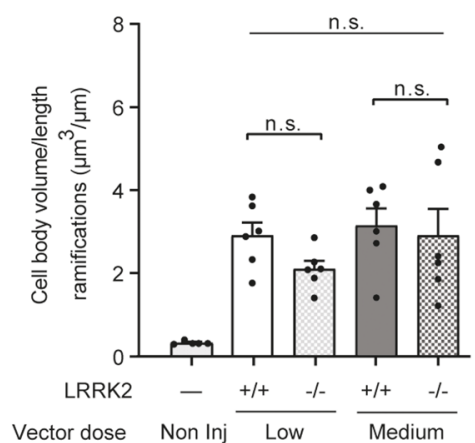

d

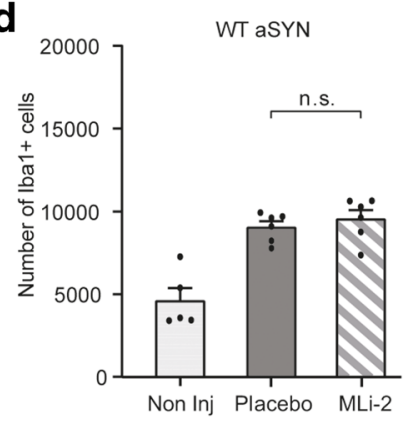

g

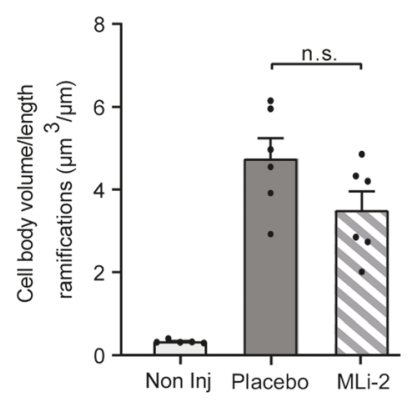

Fig. 4 Loss of LRRK2 attenuates $\alpha \mathrm{SYN}$-induced microglial activation. (A) Representative images of immunostaining for Iba1 in the SN of WT, LRRK2 KO, and MLi-2-treated animals injected with different doses of rAAV2/7 A53T or WT $\alpha$ SYN vector. Scale bars $=250 \mu \mathrm{m}$ (main picture) or $25 \mu \mathrm{m}$ (inset). (B, C) Number of $\mathrm{Iba1}^{+}$microglia in the SN of WT and LRRK2 KO rats injected with different doses of rAAV2/7 A53T (B) or WT (C) $\alpha$ SYN vector (mean \pm S.E.M., two-way ANOVA and Sidak's post hoc test, $n=6$ animals/group, $F(6,33)=4.929, p>0.05$ (B); $F(4$, $10)=0.8890, p=0.05(\mathrm{C}))$. (D) Number of $\mathrm{Ibal}^{+}$microglia in the $\mathrm{SN}$ of WT rats injected with a medium dose of rAAV2/7 WT $\alpha$ SYN and treated with MLi-2 inhibitor or placebo (mean \pm S.E.M., $t$ test, $n=6, F(2,14)=$

\section{Discussion}

Using a set of viral vector-based $\alpha \mathrm{SYN}$ rat models, we demonstrate a significant role for LRRK2 in $\alpha \mathrm{SYN}$-induced neuroinflammation, but a lack of protection against A53T or WT $\alpha \mathrm{SYN}$-induced neurodegeneration and neuropathology upon reduced LRRK2 activity. Indeed, genetic LRRK2 ablation or pharmacological LRRK2 kinase inhibition did not alter or
23.22, $p>0.05$ ). (E, F) Stereological analysis of microglial morphology in the SN of WT and LRRK2 KO rats injected with rAAV2/7 A53T or WT $\alpha$ SYN vectors. Data are presented as the ratio of mean cell body volume/length of ramifications (mean \pm S.E.M., two-way ANOVA and Sidak's post hoc test, $n=6$ animals/group; WT versus LRRK2 KO: $F(6$, $33)=2847, * * p=0.0093(\mathrm{E}) ; F(4,23)=2.146, p>0.05(\mathrm{~F})$; vector dose

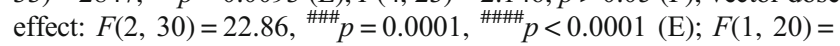
$1.530, p>0.05(\mathrm{~F}))$. (G) Stereological analysis of microglial morphology in the SN in WT rats injected with rAAV2/7 WT $\alpha \mathrm{SYN}$ (medium dose) and treated with MLi-2 or placebo (mean \pm S.E.M., $t$ test, $n=6, F(5,5)=$ $1.204, p>0.05$ )

even increased motor deficits after nigral injection of different doses of rAAV2/7 WT or A53T $\alpha$ SYN. Also, dopaminergic neurodegeneration in the $\mathrm{SN}$ and the striatal terminals was not ameliorated in conditions of reduced LRRK2 protein and/or kinase activity, with a trend towards increased neurotoxicity in LRRK2 $\mathrm{KO}$ rats. These histological observations were reinforced by Western blot analysis. Further, we show that genetic ablation or pharmacological LRRK2 inhibition did not 
a

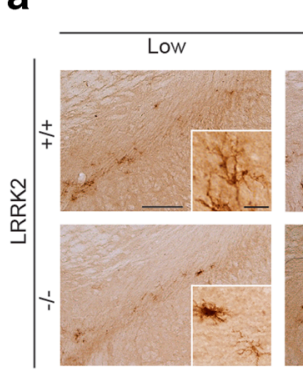

A53T aSYN

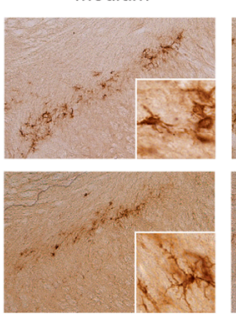

A53T aSYN
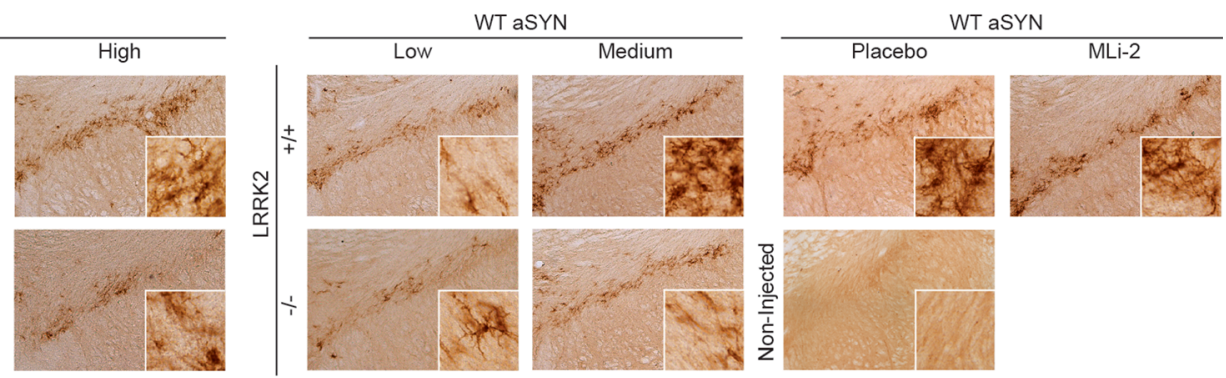

b

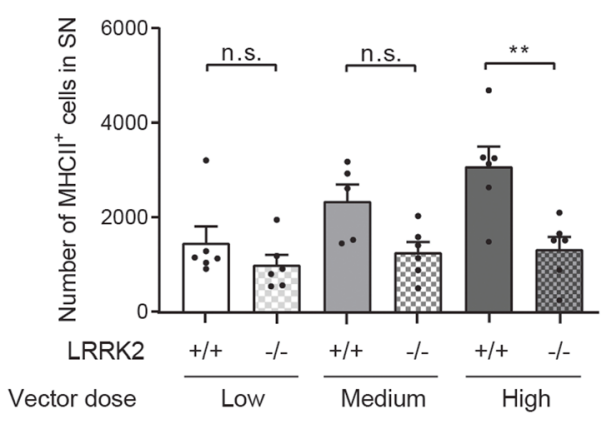

c

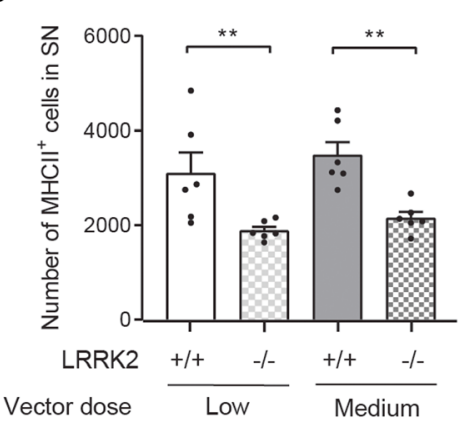

d

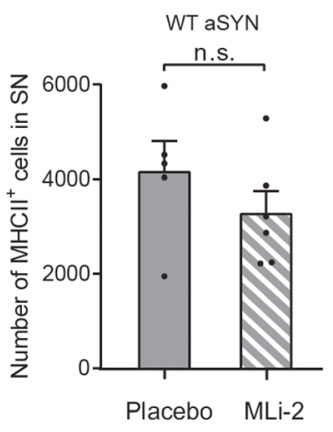

e
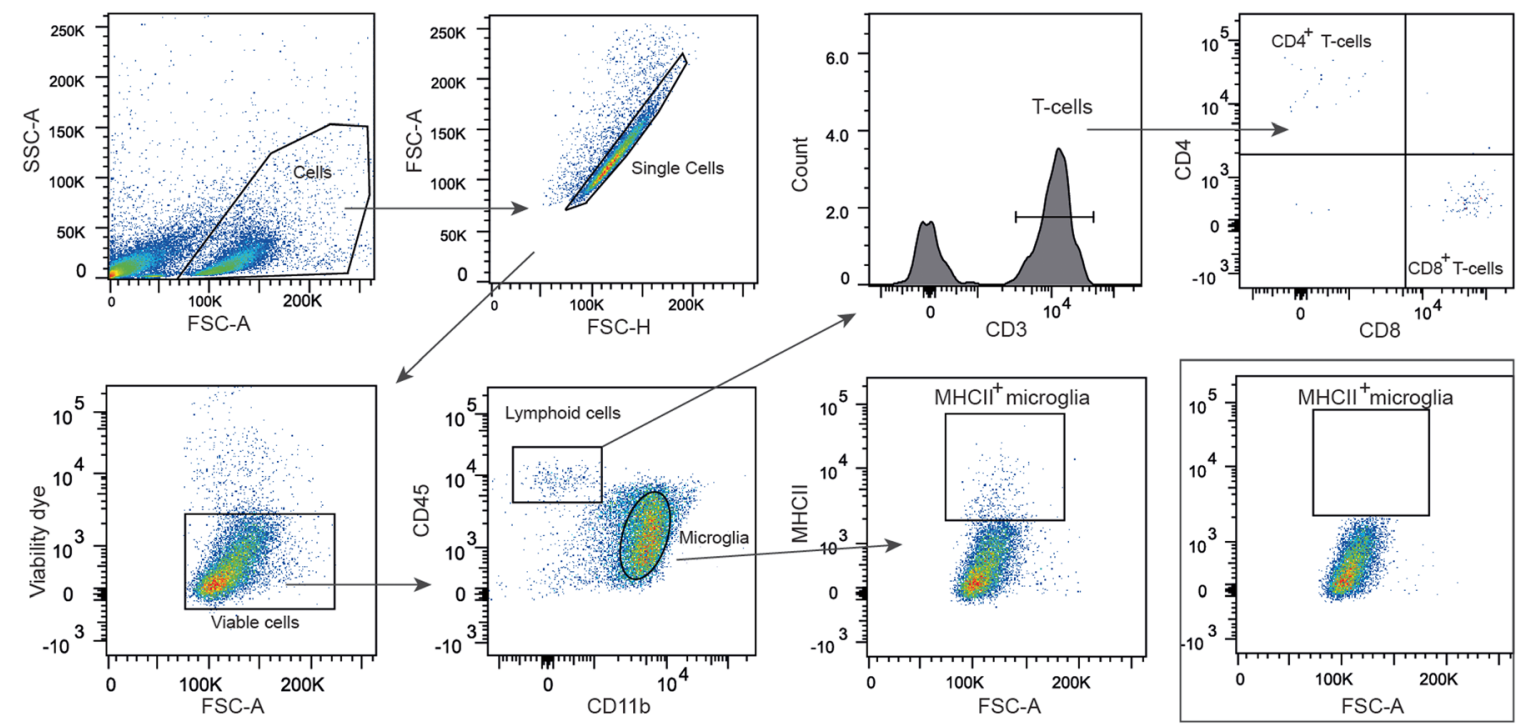

f

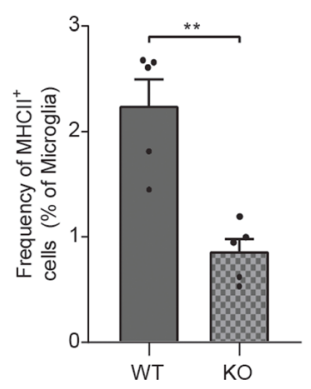

g

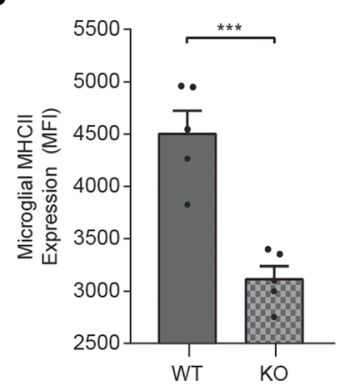

h

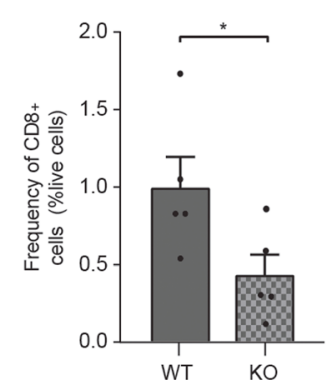

MHCII FMO control

i

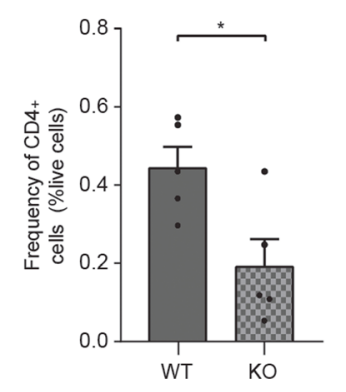

alter $\alpha$ SYN pathology in any of the models tested, suggesting that LRRK2 does not interfere with the process of $\alpha \mathrm{SYN}$ aggregation. In contrast, our findings suggest a role for LRRK2 in $\alpha \mathrm{SYN}$-mediated neuroinflammation, given the 
4 Fig. 5 Loss of LRRK2 attenuates $\alpha \mathrm{SYN}$-induced microglial MHC II activation and $\mathrm{T}$ cell infiltration. (A) Representative image of the MHC II immunostaining in the SN of WT, LRRK2 KO, and MLi-2-treated animals injected with different doses of rAAV2/7 A53T or WT $\alpha \mathrm{SYN}$ vector (scale bars $=250 \mu \mathrm{m}$ (main picture), $50 \mu \mathrm{m}$ (inset)). (B, C) Number of MHC II ${ }^{+}$cells in the SN of WT and LRRK2 KO rats injected with different doses of rAAV2/7 A53T (B) or WT (C) $\alpha$ SYN vector (mean \pm S.E.M., two-way ANOVA and Sidak's post hoc test, $n=6$ animals/group, $F(1,29)=18.33$, ** $p=0.0012$ for A53T high vector dose (B); $F(1,20)=22.27, * * p=0.0093$ for WT low vector dose, $* * p=$ 0.0046 for WT medium vector dose (C)). (D) Number of $\mathrm{MHC} \mathrm{II}^{+}$cells in the SN of WT rats injected with a medium dose of rAAV2/7 WT $\alpha \mathrm{SYN}$ and treated with MLi-2 inhibitor or placebo (mean \pm S.E.M., $t$ test, $n=6, F(4,5)=1.545, p>0.05)$. (E-I) Flow cytometry immunophenotyping on brain tissue of LRRK2 $\mathrm{KO}$ and WT rats 2 weeks after injection with a high dose of rAAV2/7 A53T $\alpha \mathrm{SYN}$ vector. (E) Gating strategy for the identification of $\mathrm{MHC}^{+}{ }^{+}$microglia, $\mathrm{CD}^{+} \mathrm{T}$ helper cells, and $\mathrm{CD} 8^{+}$cytotoxic T cells in the midbrain of LRRK2 $\mathrm{KO}$ and WT control animals. (F) Analysis of the number of $\mathrm{MHC} \mathrm{II}^{+}$microglia in LRRK2 KO and WT rats. (G) Analysis of the mean fluorescence intensity (MFI) of MHC II in microglia from LRRK2 KO and WT rats. Frequency of $\mathrm{CD}^{+}$cytotoxic $\mathrm{T}$ cells $(\mathrm{H})$ and $\mathrm{CD}^{+} \mathrm{T}$ helper cells (I) in the midbrain of LRRK2 $\mathrm{KO}$ and WT animals injected with rAAV2/7 A53T $\alpha$ SYN (mean \pm S.E.M., $t$ test, $n=5$ animals/group, $F(4,4)=$ $4.379, * * p=0.0012(\mathrm{~F}) ; F(4,4)=3.321, * * * p=0.0005(\mathrm{G}) ; F(4,4)=$ $\left.2.383, * p=0.0472(\mathrm{H}) ; F(4,4)=1.651,{ }^{*} p=0.0196(\mathrm{I})\right)$

significant reduction in the frequency of reactive microglia and infiltrated $\mathrm{CD} 4^{+}$and $\mathrm{CD} 8^{+} \mathrm{T}$ cells in the absence of LRRK2.

With LRRK2 kinase inhibitors currently in clinical studies, the question whether patients with idiopathic PD would benefit from reduced LRRK2 activity is timely and highly relevant. Using different $\alpha$ SYN-based PD models, several groups have studied the impact of reduced LRRK2 levels and/or activity, which resulted in conflicting data. Genetic ablation of LRRK2 was reported to reduce $\alpha \mathrm{SYN}$ aggregation in a transgenic mouse model overexpressing A53T $\alpha \mathrm{SYN}$ in the forebrain [10]; however, this protective effect could not be replicated in a comparable model with hindbrain overexpression [16]. Also in viral vector-based $\alpha$ SYN PD rat models, loss of LRRK2 was reported to result in either a complete rescue [11] or a lack of protection [17] against $\alpha \mathrm{SYN}$-mediated dopaminergic toxicity. Similarly, pharmacological LRRK2 kinase inhibition was reported to protect against dopaminergic cell death in a viral vector-mediated $\alpha$ SYN PD rat model [12], while a more recent study could not replicate these findings using a similar compound [17]. Also in mice exposed to $\alpha \mathrm{SYN}$ preformed fibrils (PFFs), LRRK2 kinase inhibition was not protective [18], while in contrast, a reduction in LRRK2 protein levels by antisense oligonucleotides was reported to ameliorate the dopaminergic neuronal loss induced by $\alpha$ SYN fibrils [19]. Finally, LRRK2 kinase inhibition was reported to decrease $\alpha \mathrm{SYN}$ aggregate levels in an $\alpha \mathrm{SYN}$ transgenic mouse [30] and a more recent study showed that G2019S transgenic mice exhibit higher $\alpha$ SYN PFF-induced pathology in some regions, yet lower pathology in other brain regions [31]. Altogether, these data suggest that the observed protective effect of LRRK2 deletion/inhibition might depend on the model and readout used. Therefore, with the current study, we aimed to resolve this question by an extensive characterization of both genetic and pharmacological LRRK2 inhibition in a set of robust and well-characterized $\alpha$ SYN-based models, presenting modest to more severe neurodegeneration. Our results indicate that physiological WT LRRK2 does not play a crucial role in $\alpha \mathrm{SYN}$-induced behavioral deficits, neurodegeneration, or $\alpha \mathrm{SYN}$ pathology. Still, loss of LRRK2 protein aggravated the $\alpha \mathrm{SYN}$-induced decrease in nigral and striatal TH protein levels, although this was not accompanied by more severe dopaminergic cell loss. It is currently unclear how this relates to neuronal function, yet it might explain the exacerbated motor deficits observed in the LRRK2 $\mathrm{KO}$ rats (Fig. 1B). The observation that also MLi-2-treated rats display a similar reduction in striatal TH levels might point to LRRK2 dephosphorylation at S910 and/or S935 as potential underlying mechanism. In line with these data, double knockin mice carrying the S910A/S935A mutation show alterations in dopamine-regulating proteins and a significant reduction in intensity of TH immunoreactivity without dopaminergic neurodegeneration [32]. Future studies will be necessary to address how LRRK2 regulates TH protein levels and how this is related to neuronal (dys)function. Furthermore, we found that LRRK2 deletion significantly reduces the frequency of reactive microglia and infiltrating T cells in our model. Studies on inflammatory processes in rAAV-based $\alpha \mathrm{SYN}$ models point to a causal role of neuroinflammation in the pathogenesis of PD $[27,33,34]$. The involvement of LRRK2 in the infiltration of peripheral immune cells is a very relevant finding since peripheral immune responses are believed to play an important role in PD pathogenesis [35]. Indeed, infiltrated T cells have been found in the SN of patients with PD [36] and peptides derived from $\alpha \mathrm{SYN}$ can elicit an immune response from T cells obtained from patients with PD [37]. Additionally, we have shown that peripheral injection of $\alpha \mathrm{SYN}$ fibrillar assemblies in naive mice primed with LPS resulted in differential recruitment of $\mathrm{T}$ cells to the brain [38]. Recently, humanderived monocytes have been shown to react distinctly to different $\alpha$ SYN assemblies [39]. In addition, being highly expressed in myeloid cells and upregulated upon inflammatory insults [40], LRRK2 might be another important player in the immune cascade involved in the pathogenesis of PD. Multiple lines of evidence point to a significant role for LRRK2 in neuroinflammation, which is strongly believed to be, at least partly, mediated by its role in peripheral immune cells (reviewed in the study of Cabezudo et al. [35]).

The data presented here point to a role for LRRK2 in both the innate and adaptive immune responses. Remarkably, despite the suppressed microglial activation and $\mathrm{T}$ cell infiltration in the absence of LRRK2, no protection against $\alpha \mathrm{SYN}$ induced toxicity was established. This is an intriguing finding 
that underscores the complex relation between LRRK2 and $\alpha \mathrm{SYN}$ and their role in neuroinflammation and neurodegeneration. Although the animal models used in this study present high construct, face, and predictive validity, the complexity and the chronic character of these processes in the patient brain might not be fully captured.

In conclusion, our study has revealed a significant role for LRRK2 in $\alpha \mathrm{SYN}$-induced neuroinflammation. The finding that targeting LRRK2 did not protect against neurodegeneration and neuropathology should be well considered in light of the development of LRRK2 kinase inhibitors for idiopathic PD. The currently ongoing clinical studies will reveal the true therapeutic potential of LRRK2 kinase inhibitors for both patients with genetic LRRK2 PD and patients with idiopathic PD. Future research is crucial to better understand the following: 1) how neuroinflammation links to the progression of PD, 2 ) to what extent $\alpha \mathrm{SYN}$ and LRRK2 are key players in these pathological processes, and 3) the timing of these processes in the progression of $\mathrm{PD}$.

Supplementary Information The online version contains supplementary material available at https://doi.org/10.1007/s13311-021-01007-8.

Acknowledgements We thank A. Aertgeerts, J. Van Asselberghs, and S. Croes for their technical assistance; the Leuven Viral Vector Core (LVVC) for the rAAV vector production; and the VIB-KU Leuven FACS Core Facility.

Required Author Forms Disclosure forms provided by the authors are available with the online version of this article.

Author Contribution AVDP coordinated, designed, and conducted part of the in vivo experiments. DC performed part of the in vivo experiments. GG helped with the behavioral analysis. JPR provided guidance and helped in analyzing the flow cytometry data. CVH was responsible for the rAAV production. VB coordinated the project and revised the manuscript. EL coordinated and designed the experiments and performed the part of the biochemical analyses. AVDP, EL, and DC wrote the manuscript. All authors approved the final manuscript.

Funding This work was supported by the Michael J. Fox Foundation, FWO Flanders (Projects G.0927.14, G080517N, G0E1917N, S006617N, and postdoctoral fellowship to A. Van der Perren), the KU Leuven (OT/ 14/120, C14/18/102), and the Medical Foundation Queen Elisabeth (PhD fellowship G. Gelders).

\section{Declaration}

Competing Interests The authors declare no competing interests.

\section{References}

1. Monfrini E, Di Fonzo A. Leucine-Rich Repeat Kinase (LRRK2) Genetics and Parkinson's Disease. Adv Neurobiol. 2017;14:3-30.

2. West AB, Moore DJ, Biskup S, et al. Parkinson's disease-associated mutations in leucine-rich repeat kinase 2 augment kinase activity. Proceedings of the National Academy of Sciences of the United States of America. 2005;102(46):16842-7.
3. Greggio E, Jain S, Kingsbury A, et al. Kinase activity is required for the toxic effects of mutant LRRK2/dardarin. Neurobiol Dis. 2006;23(2):329-41.

4. Di Maio R, Hoffman EK, Rocha EM, et al. LRRK2 activation in idiopathic Parkinson's disease. Sci Transl Med. 2018;10(451).

5. West AB. Achieving neuroprotection with LRRK2 kinase inhibitors in Parkinson disease. Exp Neurol. 2017;298(Pt B):236-45.

6. Fell MJ, Mirescu C, Basu K, et al. MLi-2, a Potent, Selective, and Centrally Active Compound for Exploring the Therapeutic Potential and Safety of LRRK2 Kinase Inhibition. J Pharmacol Exp Ther. 2015;355(3):397-409.

7. Choi HG, Zhang J, Deng X, et al. Brain Penetrant LRRK2 Inhibitor. ACS Med Chem Lett. 2012;3(8):658-62.

8. Henderson JL, Kormos BL, Hayward MM, et al. Discovery and preclinical profiling of 3-[4-(morpholin-4-yl)-7H-pyrrolo[2,3d]pyrimidin-5-yl]benzonitrile (PF-06447475), a highly potent, selective, brain penetrant, and in vivo active LRRK2 kinase inhibitor. J Med Chem. 2015;58(1):419-32.

9. Denali. http://investors.denalitherapeutics.com/news-releases/ news-release-details/denali-therapeutics-announces-positiveclinical-results-lrrk2\#ir-pages.

10. Lin X, Parisiadou L, Gu XL, et al. Leucine-rich repeat kinase 2 regulates the progression of neuropathology induced by Parkinson's-disease-related mutant alpha-synuclein. Neuron. 2009;64(6):807-27.

11. Daher JP, Volpicelli-Daley LA, Blackburn JP, Moehle MS, West AB. Abrogation of alpha-synuclein-mediated dopaminergic neurodegeneration in LRRK2-deficient rats. Proceedings of the National Academy of Sciences of the United States of America. 2014;111(25):9289-94.

12. Daher JP, Abdelmotilib HA, Hu X, et al. Leucine-rich Repeat Kinase 2 (LRRK2) Pharmacological Inhibition Abates alphaSynuclein Gene-induced Neurodegeneration. J Biol Chem. 2015;290(32):19433-44.

13. Volpicelli-Daley LA, Abdelmotilib H, Liu Z, et al. G2019SLRRK2 Expression Augments alpha-Synuclein Sequestration into Inclusions in Neurons. J Neurosci. 2016;36(28):7415-27.

14. Tsika E, Nguyen AP, Dusonchet J, Colin P, Schneider BL, Moore DJ. Adenoviral-mediated expression of G2019S LRRK2 induces striatal pathology in a kinase-dependent manner in a rat model of Parkinson's disease. Neurobiol Dis. 2015;77:49-61.

15. Bieri G, Brahic M, Bousset L, et al. LRRK2 modifies alpha-syn pathology and spread in mouse models and human neurons. Acta Neuropathol. 2019;137(6):961-80.

16. Daher JP, Pletnikova O, Biskup S, et al. Neurodegenerative phenotypes in an A53T alpha-synuclein transgenic mouse model are independent of LRRK2. Hum Mol Genet. 2012;21(11):2420-31.

17. Andersen MA, Christensen KV, Badolo L, et al. Parkinson's disease-like burst firing activity in subthalamic nucleus induced by AAV-alpha-synuclein is normalized by LRRK2 modulation. Neurobiol Dis. 2018;116:13-27.

18. Henderson MX, Sengupta M, McGeary I, et al. LRRK2 inhibition does not impart protection from alpha-synuclein pathology and neuron death in non-transgenic mice. Acta Neuropathol Commun. 2019;7(1):28.

19. Zhao HT, John N, Delic V, et al. LRRK2 Antisense Oligonucleotides Ameliorate alpha-Synuclein Inclusion Formation in a Parkinson's Disease Mouse Model. Mol Ther Nucleic Acids. 2017;8:508-19.

20. Maekawa T, Sasaoka T, Azuma S, et al. Leucine-rich repeat kinase 2 (LRRK2) regulates alpha-synuclein clearance in microglia. BMC Neurosci. 2016;17(1):77.

21. Russo I, Kaganovich A, Ding J, et al. Transcriptome analysis of LRRK2 knock-out microglia cells reveals alterations of inflammatory- and oxidative stress-related pathways upon treatment with alpha-synuclein fibrils. Neurobiol Dis. 2019;129:67-78. 
22. Oliveras-Salva M, Van der Perren A, Casadei N, et al. rAAV2/7 vector-mediated overexpression of alpha-synuclein in mouse substantia nigra induces protein aggregation and progressive dosedependent neurodegeneration. Mol Neurodegener. 2013;8:44.

23. Van der Perren A, Toelen J, Casteels C, et al. Longitudinal followup and characterization of a robust rat model for Parkinson's disease based on overexpression of alpha-synuclein with adeno-associated viral vectors. Neurobiol Aging. 2015;36(3):1543-58.

24. Van der Perren A, Toelen J, Carlon M, et al. Efficient and stable transduction of dopaminergic neurons in rat substantia nigra by $\operatorname{rAAV} 2 / 1,2 / 2,2 / 5,2 / 6.2,2 / 7,2 / 8$ and $2 / 9$. Gene Ther. 2011;18(5):517-27.

25. Baekelandt V, Claeys A, Eggermont K, et al. Characterization of lentiviral vector-mediated gene transfer in adult mouse brain. Hum Gene Ther. 2002;13(7):841-53.

26. Schmitz C, Hof PR. Design-based stereology in neuroscience. Neuroscience. 2005;130(4):813-31.

27. Van der Perren A, Macchi F, Toelen J, et al. FK506 reduces neuroinflammation and dopaminergic neurodegeneration in an alphasynuclein-based rat model for Parkinson's disease. Neurobiol Aging. 2015;36(3):1559-68.

28. Dzamko N, Deak M, Hentati F, et al. Inhibition of LRRK2 kinase activity leads to dephosphorylation of $\operatorname{Ser}(910) / \operatorname{Ser}(935)$, disruption of 14-3-3 binding and altered cytoplasmic localization. Biochem J. 2010;430(3):405-13.

29. Anderson JP, Walker DE, Goldstein JM, et al. Phosphorylation of Ser-129 is the dominant pathological modification of alphasynuclein in familial and sporadic Lewy body disease. J Biol Chem. 2006;281(40):29739-52.

30. Bae EJ, Kim DK, Kim C, et al. LRRK2 kinase regulates alphasynuclein propagation via RAB35 phosphorylation. Nat Commun. 2018;9(1):3465.

31. Henderson MX, Cornblath EJ, Darwich A, et al. Spread of alphasynuclein pathology through the brain connectome is modulated by selective vulnerability and predicted by network analysis. Nat Neurosci. 2019;22(8):1248-57.
32. Zhao Y, Keshiya S, Atashrazm F, et al. Nigrostriatal pathology with reduced astrocytes in LRRK2 S910/S935 phosphorylation deficient knockin mice. Neurobiol Dis. 2018;120:76-87.

33. Sanchez-Guajardo V, Febbraro F, Kirik D, Romero-Ramos M. Microglia acquire distinct activation profiles depending on the degree of alpha-synuclein neuropathology in a rAAV based model of Parkinson's disease. PLoS One. 2010;5(1):e8784.

34. Harms AS, Thome AD, Yan Z, et al. Peripheral monocyte entry is required for alpha-Synuclein induced inflammation and Neurodegeneration in a model of Parkinson disease. Exp Neurol. 2018;300:179-87.

35. Cabezudo D, Baekelandt V, Lobbestael E. Multiple-hit hypothesis in Parkinson's disease: LRRK2 and inflammation. Frontiers in Neuroscience. 2020;14:376.

36. Brochard V, Combadiere B, Prigent A, et al. Infiltration of $\mathrm{CD} 4^{+}$lymphocytes into the brain contributes to neurodegeneration in a mouse model of Parkinson disease. J Clin Invest. 2009;119(1):182-92.

37. Sulzer D, Alcalay RN, Garretti F, et al. T cells from patients with Parkinson's disease recognize alpha-synuclein peptides. Nature. 2017;546(7660):656-61.

38. Peralta Ramos JM, Iribarren P, Bousset L, Melki R, Baekelandt V, Van der Perren A. Peripheral Inflammation Regulates CNS Immune Surveillance Through the Recruitment of Inflammatory Monocytes Upon Systemic alpha-Synuclein Administration. Front Immunol. 2019;10:80.

39. Grozdanov V, Bousset L, Hoffmeister M, et al. Increased Immune Activation by Pathologic alpha-Synuclein in Parkinson's Disease. Ann Neurol. 2019;86(4):593-606.

40. Moehle MS, Webber PJ, Tse T, et al. LRRK2 inhibition attenuates microglial inflammatory responses. J Neurosci. 2012;32(5):1602-11.

Publisher's Note Springer Nature remains neutral with regard to jurisdictional claims in published maps and institutional affiliations. 